\title{
Humidity Calibration Automation
}

\author{
Fulvio FENOTTI ${ }^{* *}$ and David VASTY2 ${ }^{2 *}$ \\ ${ }^{1}$ TRESCAL, Lab Department, Via dei Metalli, 1, 25039 Travagliato, ITALY \\ ${ }^{2}$ TRESCAL, Group Technical Department, 9 rue Alfred Kastler, , Rouen, FRANCE
}

\begin{abstract}
This article describes an automated humidity calibration system developed in Trescal Italy using a software developed internally, a high resolution Digital Single-Lens Reflex (DSLR) camera, climatic chambers and chilled mirror hygrometers as reference standards.
\end{abstract}

\section{Introduction}

The calibration of thermos-hygrometer instrumentation is a time consuming long run processing which the environment humidity and temperature are artificially forced to specific values.

The calibration process usually involves the use of dedicated climatic chamber machines capable to modify environmental conditions. Once the chamber is loaded with the units under test to calibrate, a required air temperature and air humidity values are set in the chamber controller and the process begins.

Once the chamber reaches the desired set point and show a stable behavior around this one, the comparative measure is done simultaneously reading the value of one or more reference standards for the current air temperature and humidity level and reading each unit under test output values.

The whole process is a time consuming task for the lab personnel, requiring constant attention to properly check the process step, special attention has to be paid particularly during stability points because several simultaneously reading from different instruments have to be taken in order to properly evaluated the reach of stability.

An automation system capable to reproduce all required steps for drive the entire process and perform a good data acquisition can drastically increase the productivity of the lab division, introduce a better uniformity in the used method to operate with and relief lab personnel from the long process pollings.

\section{Automation overview [1]}

The automation developed in Trescal lab was mainly based on the concept of distributed system, in which a set of independent nodes cooperate to aim for a common goal.

\footnotetext{
${ }^{*}$ Corresponding author: fulvio.fenotti@trescal.com, david.vasty@trescal.com
} 
Each node involved in the system are designed to perform a specific task and to cooperate with the others using a message passing based communication mechanism in order to supply a specific service. The automation uses this kind of architecture to control the whole data acquisition process in a non supervisionated mode but at the same time ensuring that the acquired data are compliance to the laboratory's procedures.

Currently automation runs on top of a GNU/Linux system and the software was entirely developed using the Python [2] language to be easily compatible and ported to several different operating systems and architectures. The communication mechanism is based on asynchronous events and uses the ZeroMQ as message passing framework.

The actual system controls two climatic chambers, a Votsch VC 4018 and VC3 4018 and uses two Mitchell Optidew chilled mirror hygrometers as reference standards for both air temperature and dewpoint.

\section{Calibration process definition}

Due to a numerous different factors, sometimes a specific behavior have to be taken in the calibration process (i.e. specific settings for reaching stability in extreme climatic conditions).

For this purpose the system allows definition of the full calibration process through a minimal dedicated set of procedural statements.

Although a general purpose settings are always loaded during system bootstrap, a large set of parameters and available features can be controlled and modified in the process definition stage.

The procedural language is also used to load the set points that have to be performed by the system, describing in this way the whole calibration process.

All the interaction with the user takes place through web technologies allows an easily control from dislocated working places without the need of additional software.

\section{System overview}

\subsection{Software general description}

The provided software consists in monolithic executable program intended to use in unsupervised humidity calibration measurements for autonomous process control and data acquisition.

The fine-grained control process is performed by the software, creating dedicated parallel processes to designed specific tasks. During the calibration routine, the software is also able to interact with the involved physical devices, such as reference measurement instruments and climatic chambers.

The software provides better adaptation to complex existing topology architectures. It is also possible to run the software over several interconnected nodes, delegating a specific node to act as a reference or a control. The overall process is performed and synchronized among the distributed nodes by a message passing mechanism.

Finally measurements and data acquisition values are stored in a separate database and exposed to the user by a powered web technology interface. 


\subsection{Description of the system}

The system is composed by the following subsystems:

1. Convergence and stability process;

2. Standard reference data acquisition and transportation;

3. Camera image capture layer;

4. Relational DataBase Management System (RDBMS) for data storage;

5. Web Server for user GUI and configuration tools.
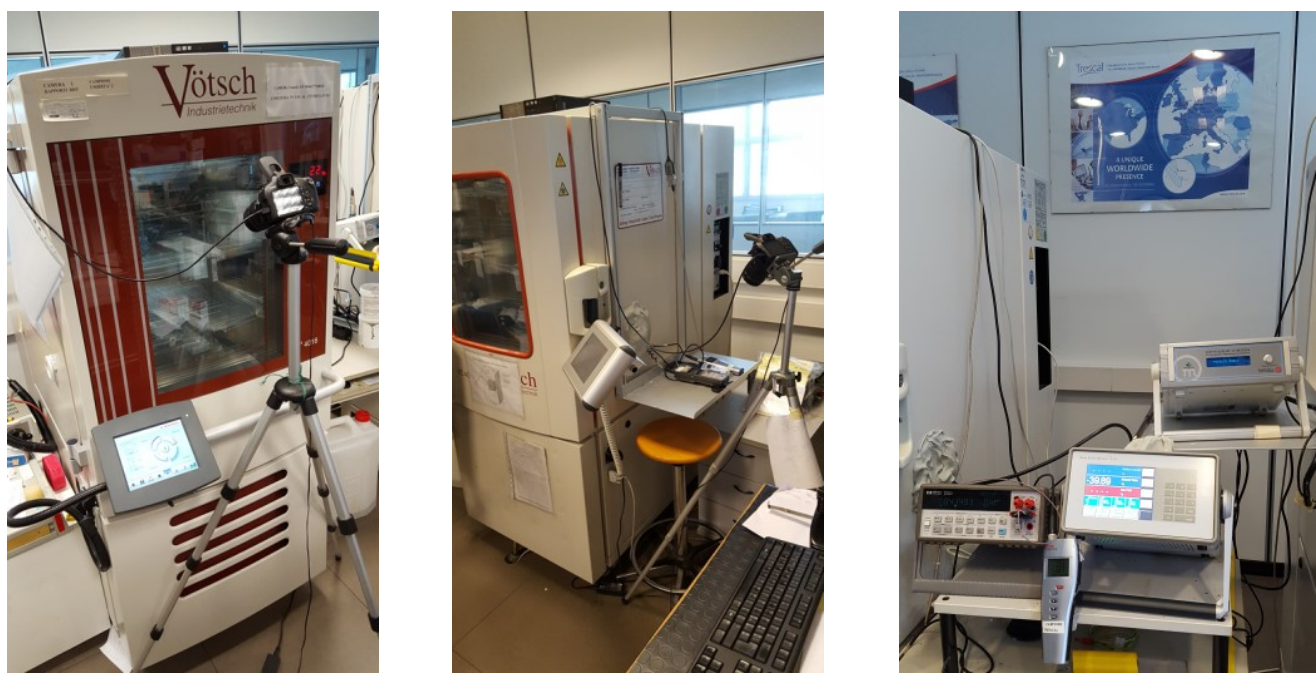

Fig. 1. The complete system: climatic chamber + camera + dew point standard

\subsubsection{Convergence and Stability process}

The stability of a measurement process is a situation in which the nature of involved physical dimensions match previous defined criteria for a certain amount of time.

In order to keep the overall process time as short as possible, but long enough to permit that the units under test can adjust themselves to the new environmental conditions, the recognition of the stability is not a trivial task. Furthermore the stability have to be held to all the needed time during data acquisition in order to get suitable measure values.

The automation uses data of measured air temperature and dew point coming from the reference standards to feed up a multistage stability algorithm capable to recognize when the environmental conditions inside the climatic chamber are suitable and starts the measure phase.

During all the time needed from the acquisition subsystem to acquire all requested data, the environmental conditions are constantly monitored to ensure soundness of the calibration procedure.

The automation uses a cooperative mechanism to synchronize the monitoring phase with the acquisition operations. If a perturbation in the stability conditions is detected during the measuring stage, all the collected data are discarded and the system partially restarts the stability matching process waiting for better stability temporal window. 


\subsubsection{Standard reference data acquisition and transportation}

In order to control this process, the main software can use data stream from some internal chamber sensors or directly from the reference standard stated in the calibration procedure.

The reference standard and the climatic chamber are connected to the computer who runs the software and drive the camera. The software could be used to perform connections to the remote devices using serial protocol such RS232 or Ethernet protocols.

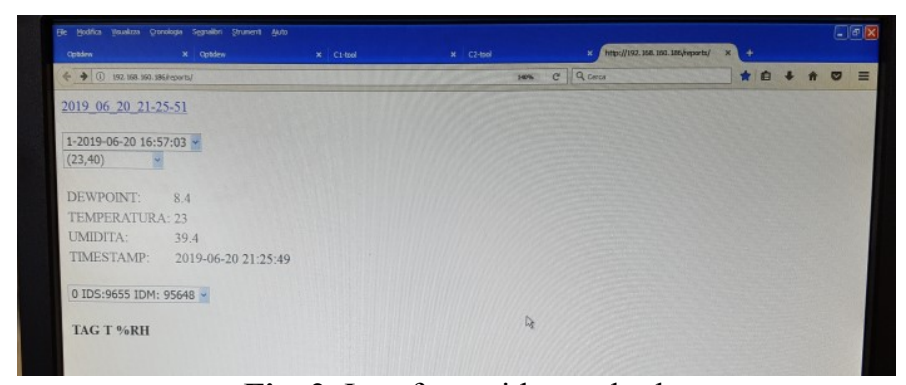

Fig. 2. Interface with standard

\subsubsection{Camera image capture layer}

The software dialogues with a high resolution Digital Single-Lens Reflex (DSLR) camera to take the pictures of the display or the analogical indicator of the instruments placed either internally or externally to the climatic chamber. Along with the camera, the use of the following set of accessories is highly suggested:

A compatible power adapter kit, to power the camera directly from an AC main power grid socket, providing a stable and durable power source

An 8 or 16 Gbyte Micro SD card with compatible adaptor, in order to provide an available memory where to store photos locally on the camera avoiding data losing in blackout situations

$>$ A long enough (typically $3 \mathrm{~m}$ or $5 \mathrm{~m}$ ) micro USB cable to directly plug the camera to the control computer

$>$ A stable and tall enough tripod to point the camera (not required for testing).

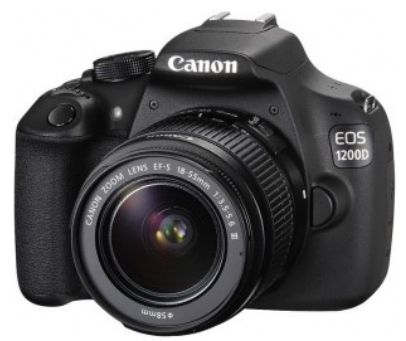

Fig. 3. Camera used for taking photos of thermos-hygrometers to calibrate 


\subsubsection{Relational DataBase Management System (RDBMS) for data storage}

All measurement points are formed by ten single measures; both the data of the dew points and the photos taken by the camera subsystem are stored in a database and can be accessed remotely at any time.

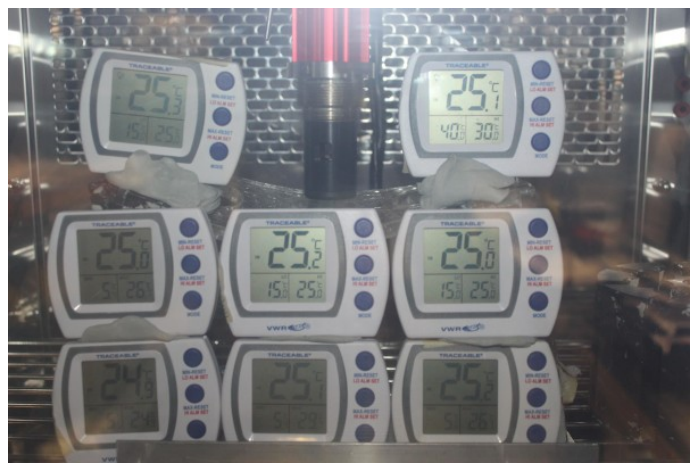

Fig. 4. A sample photo with the climatic chamber loaded with multiple thermo-hygrometers

\subsubsection{Web Server for user GUI (Graphical User Interface) and configuration tools}

The graphical user interface needed to the loading stage and to view the data is provided through a web server, usually installed on the same computer on which runs the main application program.

Real-time multi-user access is provided to each running instance node, the user can gain access to the graphical interface through a regular web browser from several different clients anywhere inside the same computers network.

Each running node instance provides a web service exposing a graphic user interface for direct node processes control. Depending on the node role, users will be able to directly interact with the provided node capabilities.

Calibration set points and others required parameters can be specified by the user using a simple short procedural language notation.

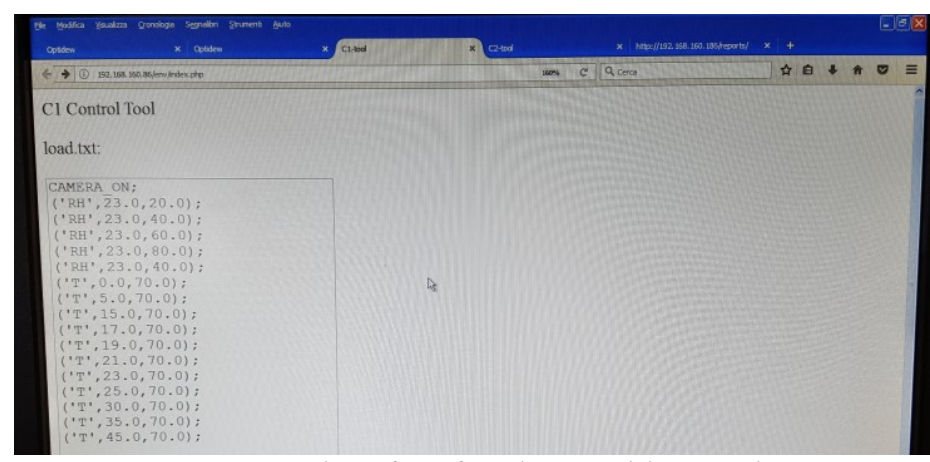

Fig. 5. User interface for photos with set points

At the end of the whole calibration process, all the acquired data, measurements and UUT photos will be available to the final user by a dedicated database interaction interface. 


\section{Results}

Currently the automation system has been using for more than four years in Trescal lab for a total of about 2000 cycles, with a very small rejected or failed portion of less than $1 \%$ and with an overall reduction of more than $50 \%$ of the working efforts needed for this particular task.

Furthermore the system was also useful to discover incoherence of the measurement values due to a shift in the climatic chamber behaviour caused to an internal component failure.
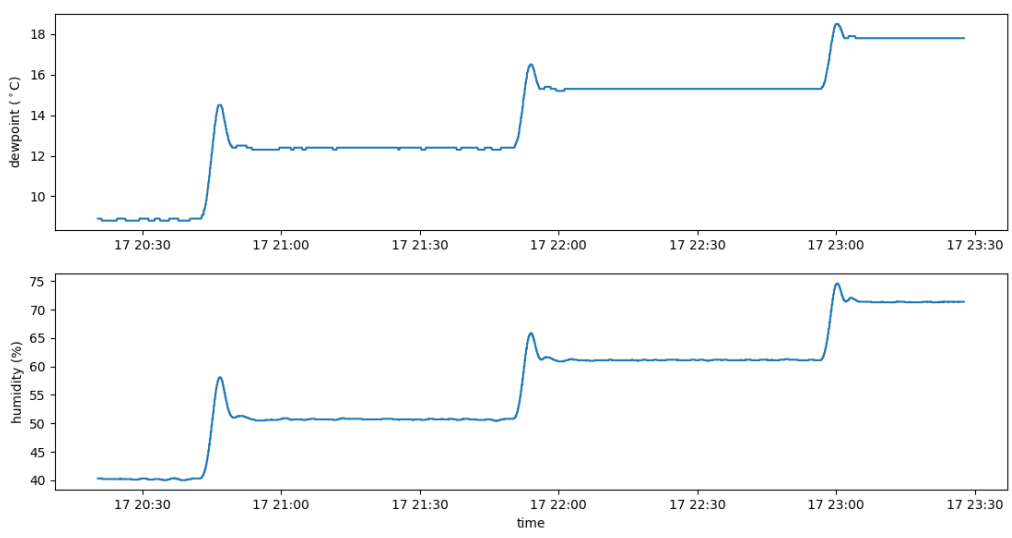

Fig. 6. Example of typical calibration process

\section{Conclusion}

A Trescal automation in the humidity calibration process has been presented, results coming from a long term use, show the soundness of the developed system and prove the enhancement of the Trescal lab production capacity, conferring an overall lab division throughput and extending the available working time to other simultaneous production activity.

Furthermore the system has been able to ensure a uniform criteria for the stability point recognition, permitting to achieve a uniform and standardized procedural method compared to personnel employment one in resulting to a better quality in the calibration process.

We decided to install this solution with improvement of the software in Trescal Group $\left(1^{\text {st }}\right.$ step in France and in a $2^{\text {nd }}$ step in Trescal Labs where we calibrate in Humidity).

At last, future developments can include planning algorithm for automatic generation of the overall process set points displacement from single unit under test set points definitions for total process time reduction and introduction of statistical models for reduction in acquisition data rejection due to display digit noise. 


\section{References}

1. A. MUSATTI, Technical Reference Note (2019)

2. https://www.python.org 